\title{
Modified 1/N Expansion for the Dirac Equation for Screened Coulomb Potential
}

\author{
Rajkumar Roychoudhury \\ Physics and Applied Mathematics Unit, Indian Statistical Institute, Calcutta 700035, India
}

Swati Panchanan

Ananda Ashram Sarada Vidyapeeth, 104, Barrackpore Trunk Road, Calcutta 700035, India

Z. Naturforsch. 48a, 1081 -1085 (1993); received April 24, 1993

It is shown that if one chooses the shift parameter correctly then the lowest order term in the modified $1 / N$ expansion for the Dirac equation as formulated by Stepanov and Tutik gives accurate results for Coulomb like potentials. Explicit eigenvalues have been obtained for the screened Coulomb potential

$$
V(r)=-\frac{v_{0}}{r}\left[1-r \lambda\left(1-\frac{1}{z}\right) /(1+r \lambda)\right],
$$

and these are compared with exact numerical results and the results obtained by the choice of shift suggested by Stepanov and Tutik.

Recently Stepanov and Tutik [1] proposed a new approach to the relativistic $1 / N$ expansion by applying the method of $h$ expansion. They argued that this method resolves the difficulties associated with the previous attempts [2-5] to apply the shifted $1 / N$ method to the Dirac equation. Since most of the previous attempts made some assumptions to reduce the Dirac equation to a Schrödinger type equation with energy dependent potential [6], not only the lowest order $1 / N$ result does not reproduce the exact Coulomb result but the full $1 / N$ expansion too.

Though the method proposed by Stepanov and Tutik reproduced the perturbation series for the Coulomb result, it has the same limitation as the previous attempts, viz. the lowest order term, unlike the Schrödinger case does not reproduce the Coulomb result. However, if one takes the choice of the shift parameter in a manner similar to that used for the Schrödinger equation $[7,8]$ it can be shown that the lowest order energy term obtained by the method of reference [1] reproduces the coupling constant expansion upto the sixth order.

In this paper we use this criterion to determine the shift parameter and show explicitly for the Dirac

Reprint requests to Prof. R. Roychoudhury, Physics and Applied Mathematics Unit, Indian Statistical Institute, 203 Barrackpore Trunk Road, Calcutta 700035, India.
Coulomb potential that $E_{0}$ gives correctly terms upto at least sixth order in the coupling constant. We then apply this method to a screened Coulomb potential of the form

$$
V(r)=-\frac{v_{0}}{r}\left[1-r \lambda\left(1-\frac{1}{z}\right) /(1+r \lambda)\right],
$$

which has been studied by several authors [9-11]. Recently, Panja et al. [12] studied this potential in the framework of a spin dependent KG equation. To simulate the effect of spin on the relativistic motion of the particle a spin dependent term is included in this $\mathrm{KG}$ framework.

However, we think the spin $1 / 2$ particle should be treated in the Dirac frame if possible, and Stepanov and Tutik have shown that this can be done and one can calculate corrections upto any order albeit it needs rather involved algebra for large order corrections. In this paper we suggest another choice of the shift parameter in addition to [1], which gives zero for the first order correction.

The essential steps of the modified $1 / N$ relativistic approximation as formulated in [1] which are relevant for the present work are given below. The radial part of the Dirac equation with a vector potential $V(r)$ and a scalar potential $m(r)$ can be written as

$$
\hbar \frac{\mathrm{d} F}{\mathrm{~d} r}-\frac{h \varkappa}{r} F(r)=(V(r)+m(r)-E) G(r)
$$

0932-0784 / $93 / 1100-1081 \$ 01.30 / 0$. - Please order a reprint rather than making your own copy. 
and

$$
\hbar \frac{\mathrm{d} G}{\mathrm{~d} r}+\frac{h \varkappa}{r} G(r)=(E-V(r)+m(r)) F(r)
$$

where $x=\frac{s}{2}\left(N_{j}-2\right), N_{j}=N+2 j$ and $s= \pm 1$.

The total angular momentum $j$ is given by $j=l-\frac{s}{2}$. Equations (1) and (2) can be reduced to a Riccati like equation of the form

$$
\begin{aligned}
& \begin{aligned}
\hbar \frac{\mathrm{d} C}{\mathrm{~d} r}(r)-\hbar Q(r) C(r)+C^{2}(r) & \\
=\frac{(\bar{k}+\hbar \sigma)(\bar{k}+\hbar(\sigma+2 s))}{4 r^{2}} & +m^{2}(r)-[E-V(r)]^{2} \\
& +\hbar Q(r) \frac{s(\bar{k}+\hbar \sigma)}{2 r},
\end{aligned} \\
& \text { where }
\end{aligned}
$$

$Q(r)=\left(\frac{\mathrm{d} m}{\mathrm{~d} r}(r)-\frac{\mathrm{d} V}{\mathrm{~d} r}(r)\right) /(E+m(r)-\mathrm{V}(r))$

and $\bar{k}$ is given by

where

$$
\bar{k}=\hbar[N+2 j-s-a],
$$

$$
a=\sigma+2-s .
$$

Equation (3) can be solved by expanding $E, C$, and $Q$ in powers of $h$, viz.,

$E=\sum_{j=0}^{\infty} E_{j} \hbar^{j}, C(r)=\sum_{j=0}^{\infty} C_{j} \hbar^{j}, Q(r)=\sum_{j=0}^{\infty} Q_{j} \hbar^{j}$

and then equating equal powers of $\hbar$ from both sides of (3). The relevant results for our discussion are the following. For $\hbar \rightarrow 0$, the lowest order energy term is given by

$$
E_{0}=V(r)+m\left(r_{0}\right)\left(1+\frac{k^{2}}{4 r_{0}^{2} m^{2}\left(r_{0}\right)}\right)^{1 / 2},
$$

where $r_{0}$ is so chosen as to make the effective potential $\left(E_{0}\right)$ minimum, i.e. $r_{0}$ is determined from

$$
\frac{\partial E_{0}}{\partial r_{0}}=0 \text { and } \frac{\partial^{2} E}{\partial r_{0}^{2}}>0,
$$

which gives

$$
r_{0}^{3} m^{\prime}\left(r_{0}\right)+r_{0}^{3} V^{\prime}\left(r_{0}\right)\left(1+\frac{k^{2}}{4 r_{0}^{2} m^{2}\left(r_{0}\right)}\right)^{1 / 2}=\frac{\bar{k}^{2}}{4 m\left(r_{0}\right)} .
$$

Since we have two unknown parameters, viz. $\bar{k}$ (depending on the shift $a$ ) and $r_{0}$, we need another equation apart from (9). This is provided by putting con- strain on $E_{1}$, the first order energy term given by

$$
\begin{gathered}
E_{1}=\frac{1}{2\left(E_{0}-V_{0}\right)}\left(\frac{\bar{k}(\sigma+s)}{2 r_{0}^{2}}+(2 n+1) \frac{w}{r_{0}}\right. \\
\left.+\frac{s \bar{k}}{2 r_{0}^{2}} \frac{m_{1}-V_{1}}{E_{0}+m_{0}-V_{0}}\right),
\end{gathered}
$$

where $w$ is given by

$w^{2}=\frac{3 k^{2}}{4 r_{0}^{2}}+2 E_{0} V_{2}+2 m_{1} m_{2}+m_{1}^{2}-\left(2 V_{0} V_{2}+V_{1}^{2}\right)$.

$m_{0}, V_{0}, m_{1}, V$, etc., are defined by the expansions of $V$ and $m$ in the following way:

where

$$
V(x)=\sum_{i=0}^{\infty} V_{i} x^{i}, \quad m(r)=\sum_{i=0}^{\infty} m_{i} x^{i},
$$

$$
x=\frac{\left(r-r_{0}\right)}{r_{0}} .
$$

As mentioned earlier, our choice of $a$ is such that $E_{1}=0$. We know that in Schrödinger problems it exactly reproduces the harmonic oscillator and Coulomb results. We apply the same ansatz for the following cases.

\section{Coulomb Problem}

If we apply this ansatz to the Dirac-Coulomb problem with $V(r)=-\frac{\beta}{r}, m_{0}=m, m_{i}=0, i>0$, then the following values for $r_{0}, E_{0}$, and $E_{1}$ are obtained:

$$
\begin{aligned}
& r_{0}=\left(\frac{\bar{k}^{2}}{4 m \beta}\right) \sqrt{1-\frac{4 \beta^{2}}{\bar{k}^{2}}}, \\
& E_{0}=m \sqrt{1-\frac{4 \beta^{2}}{\bar{k}^{2}}},
\end{aligned}
$$

and

$$
E_{1}=\frac{4 m \beta^{2}}{\bar{k}^{3}}\left(2 n+1+\sigma+s+\frac{\sigma \times 4 \beta^{2} / \bar{k}^{2}}{\left(1-4 \beta^{2} / k^{2}\right)+\sqrt{1-4 \beta^{2} / \bar{k}^{2}}}\right) .
$$

If we choose $E_{1}=0$, then we obtain immediately from (16)

$$
-\sigma=2 \bar{n} \sqrt{1-\frac{4 \beta^{2}}{\bar{k}^{2}}},
$$

where

$$
\bar{n}=n+\frac{s+1}{2} .
$$


Replacing $\sigma$ by $2 j+1-\bar{k}$ (we take $\hbar=1$ as we are interested in expansion in terms of $\beta^{2}$ ) we get by squaring (17)

$$
(2 j+1-\bar{k})^{2}=4 \bar{n}^{2}\left(1-\frac{4 \beta^{2}}{\bar{k}^{2}}\right) .
$$

This is a 4-th degree equation in $\bar{k}$ and can be solved in terms of $\beta$. However, expanding $\bar{k}$ in terms of $\beta$ we write

$$
k=k_{0}+a_{1} \beta^{2}+a_{2} \beta^{4}+\ldots,
$$

where $k_{0}=2(\bar{n}+\chi)$, where $2 \varkappa=2 j+1$.

Putting (19) in (18) we get

$$
a_{1}=-\frac{4 \bar{n}}{k_{0}^{2}}, \quad a_{2}=-\frac{4 \bar{n}}{k_{0}^{4}}+O\left(\frac{1}{k_{0}^{5}}\right) .
$$

Now from

$$
E_{0}=m \sqrt{1-\frac{4 \beta^{2}}{\bar{k}^{2}}}
$$

we get, expanding the r.h.s. of (21) in powers of $\beta^{2} / k_{0}^{2}$ (we take $m=1$ ),

$$
E_{0} \simeq 1-\frac{2 \beta^{2}}{k_{0}^{2}}\left(1+\frac{8 \bar{n} \beta^{2}}{k_{0}^{3}}\right)-\frac{2 \beta^{4}}{k_{0}^{4}}-\frac{4 \beta^{6}}{k_{0}^{6}},
$$

where we have kept terms of order upto $\beta^{6} / k_{0}^{6}$. Now the exact analytical solution to the $N$-dimensional Dirac-Coulomb problem is given by (with $\hbar=1$ )

$$
E_{\text {Dirac }}=m\left(1+\frac{\beta^{2}}{\left[\bar{n}+\sqrt{\left(\varkappa^{2}-\beta^{2}\right)}\right]^{2}}\right)^{-1 / 2} .
$$

Writing $2(\bar{n}+\chi)=k_{0}$ and expanding the r.h.s. of (23) in powers of $\beta^{2}$, we obtain, after some algebra (taking $m=1$ ),

$$
E_{\text {Dirac }} \sim 1-\frac{2 \beta^{2}}{k_{0}^{2}}\left(1+\frac{8 \bar{n} \beta^{2}}{k_{0}^{3}}\right)-\frac{2 \beta^{4}}{k_{0}^{4}}-\frac{4 \beta^{6}}{k_{0}^{6}},
$$

which is identical with (22).

\section{Screened Coulomb Potential}

We apply the above method for the Dirac equation with

$$
V(r)=-\frac{v_{0}}{r}\left[1-r \lambda\left(1-\frac{1}{z}\right) /(1+r \lambda)\right],
$$

where $\alpha=(137.037)^{-1}, \lambda=0.98 \alpha z^{1 / 3}$ and $v_{0}=a z$ and $m_{0}=m, m_{i}=0, i>0$. Then (9) simplifies to

$$
r_{0}^{3} V^{\prime}\left(r_{0}\right)\left(1+\frac{k^{2}}{4 r_{0}^{2} m^{2}}\right)^{1 / 2}=\frac{k^{2}}{4 m},
$$

which can be solved for $k$ and gives

$$
\bar{k}=\left(2 r_{0}^{4} V^{\prime 2}\left(r_{0}\right)+2 r_{0}^{2} V^{\prime}\left(r_{0}\right) \sqrt{4 m^{2} r_{0}^{2}+r_{0}^{4} V^{\prime 2}}\right)^{1 / 2} .
$$

Equation (27) and the equation $E_{1}=0$ or,

$$
\frac{\bar{k}(\sigma+s)}{2 r_{0}}+(2 n+1) \frac{w}{r_{0}}-\frac{s \bar{k} V_{1}}{2 r_{0}^{2}\left(E_{0}+m_{0}-V_{0}\right)}=0
$$

would give $\bar{k}$ and $r_{0}$, and $E_{0}$ is then obtained from (8). In Table 1, we give the eigenvalues for several values of $Z$ running from 14 to 84 for $2 \mathrm{~S}-2 \mathrm{P}$ states both for the choice of shift suggested in the present paper and for the choice of shift suggested in [1]. It may be mentioned in this connection that in [1], two choices of $a$ were suggested, viz., (i) $a=2-s$ and (ii) $a=$ $-(2 n+2 s-1)$. Now the choice (i) always gives $\bar{k}=2$ for $j=\frac{1}{2}$ and hence cannot distinguish between the ground state $E_{1,1 / 2}^{+}$and the excited state $E_{2,1 / 2}^{+}$, which makes it unrealistic for the present calculation. Hence we restrict our choice to $a=-(2 n+2 s-1)$. This choice, however, fails to lift the degeneracy among the states $E_{2,1 / 2}^{+}, E_{1,1 / 2}^{-}$and $E_{1,3 / 2}^{-}$since in all these cases the value of $n+l$ remains the same. Since our choice is such that $E_{1}=0$, we have calculated the first order correction for the choice $a=-(2 n+2 s-1)$, and the corrected values are also shown in Table 1 (square brackets with * marks). The addition of the first order correction considerably improves the results of Stepanov and Tutik's model. However, in many cases the correction terms are high. (In one case it is almost 50 percent of the value shown against $E_{0}$.) Hence we think our approach will be convenient for estimation purposes when we limit ourselves to the lowest order energy term. In comparison with the results obtained by Panja et al. (including third order correction) our results for $E_{1,1 / 2}^{+}$and $E_{1,3 / 2}^{-}$are better. However, in the lowest order the splitting between $E_{2,1 / 2}^{+}$and $E_{1,1 / 2}^{-}$shows an error of upto 7 percent for $Z \geq 74$. For lower values of $Z$ the results are excellent. This shows that higher order calculations are necessary for $E_{2,1 / 2}^{+}$and $E_{1,1 / 2}^{-}$states. But for $E_{1,1 / 2}^{+}$and $E_{1,3 / 2}^{-}$our results are almost exact for $Z \geq 74$.

To conclude, we have suggested an alternative choice of shift in the relativistic $1 / N$ expansion method which gives quite accurate values at the lowest level and is especially suitable for modified Coulomb type potentials if one restricts oneself to the lowest order energy term. 
Table 1. Eigenvalues $E_{n j}^{\mathrm{P}}$ (in $\mathrm{keV}$ ), where P denotes parity for the potential given by (25) obtained from the modified shifted $1 / N$ expansion. Only lowest order values are given. The figures in the parenthesis and square brackets are the exact numerical values (see [11]) and the lowest order value obtained by the choice of shift taken in [1], respectively. The values within * marked square brackets are obtained by taking account of the first order correction as given in [1].

\begin{tabular}{|c|c|c|c|c|c|c|}
\hline \multirow[t]{2}{*}{$Z$} & \multicolumn{3}{|l|}{$E_{1,1 / 2}^{+}$} & \multicolumn{3}{|l|}{$E_{2,1 / 2}^{+}$} \\
\hline & $\begin{array}{l}\text { Energy } \\
\text { values }\end{array}$ & $\begin{array}{l}a^{*}=3 \\
a \text { (present } \\
\text { model) }\end{array}$ & $\begin{array}{l}\text { Percent- } \\
\text { age error }\end{array}$ & $\begin{array}{l}\text { Energy } \\
\text { values }\end{array}$ & $\begin{array}{l}a^{*}=1 \\
a \text { (present } \\
\text { model) }\end{array}$ & $\begin{array}{l}\text { Percent- } \\
\text { age } \\
\text { error }\end{array}$ \\
\hline 14 & $\begin{array}{l}-2.0012 \\
(-1.9998) \\
{[-1.9593]} \\
{[-2.0044]^{*}}\end{array}$ & 3.0158 & $0.07 \%$ & $\begin{array}{l}-0.2749 \\
(-0.2493) \\
{[-0.1870]} \\
{[-0.2791] *}\end{array}$ & 1.3342 & $10.27 \%$ \\
\hline 19 & $\begin{array}{l}-3.8692 \\
(-3.8646) \\
{[-3.8091]} \\
{[-3.8701]}\end{array}$ & 3.0122 & $0.14 \%$ & $\begin{array}{l}-0.5654 \\
(-0.5249) \\
{[-0.4237]} \\
{[-0.5756]}\end{array}$ & 1.2736 & $9.66 \%$ \\
\hline 24 & $\begin{array}{l}-6.3881 \\
(-6.3829) \\
{[-6.3133]} \\
{[-6.39908]^{*}}\end{array}$ & 3.0095 & $0.25 \%$ & $\begin{array}{l}-0.9816 \\
(-0.9268) \\
{[-0.7810]} \\
{[-0.9994]^{*}}\end{array}$ & 1.2328 & $7.83 \%$ \\
\hline 29 & $\begin{array}{l}-9.5825 \\
(-9.5740) \\
{[-9.4913]} \\
{[-9.58124]^{*}}\end{array}$ & 3.0079 & $0.08 \%$ & $\begin{array}{l}-1.5317 \\
(-1.466) \\
{[-1.2677]} \\
{[-1.5615]^{*}}\end{array}$ & 1.2038 & $4.48 \%$ \\
\hline 34 & $\begin{array}{l}-13.4668 \\
(-13.460) \\
{[-13.3639]} \\
{[-13.4675] *}\end{array}$ & 3.0065 & $0.06 \%$ & $\begin{array}{c}-2.2234 \\
(-2.151) \\
{[-1.8900]} \\
{[2.2904]^{*}}\end{array}$ & 1.1832 & $6.48 \%$ \\
\hline 39 & $\begin{array}{l}-18.0704 \\
(-18.063) \\
{[-17.9541]} \\
{[-18.0704]^{*}}\end{array}$ & 3.0054 & $0.04 \%$ & $\begin{array}{l}-3.0637 \\
(-2.993) \\
{[-2.6531]} \\
{[-3.1405]}\end{array}$ & 1.1684 & $4.93 \%$ \\
\hline 74 & $\begin{array}{l}-73.2960 \\
(-73.287) \\
{[-73.1003]} \\
{[-73.30136]^{*}}\end{array}$ & 3.0022 & $0.02 \%$ & $\begin{array}{l}-13.7337 \\
(-14.298) \\
{[-12.2675]} \\
{[-14.6858]}\end{array}$ & 1.1523 & $3.95 \%$ \\
\hline 79 & $\begin{array}{l}-85.1100 \\
(-85.101) \\
{[-84.9042]} \\
{[-85.1135]^{*}}\end{array}$ & 3.0020 & $0.01 \%$ & $\begin{array}{l}-16.0507 \\
(-16.287) \\
{[-14.2949]} \\
{[-17.3021]^{*}}\end{array}$ & 1.1579 & $1.44 \%$ \\
\hline 84 & $\begin{array}{l}-98.140 \\
(-98.145) \\
{[-97.938]} \\
{[-98.1576]^{*}}\end{array}$ & 3.0017 & $0.005 \%$ & $\begin{array}{l}-18.6034 \\
(-19.165) \\
{[-16.4969]} \\
{[-20.2219]^{*}}\end{array}$ & 1.1720 & $2.93 \%$ \\
\hline
\end{tabular}

\begin{tabular}{lllll}
\hline$Z$ & $E_{1,1 / 2}^{-}$ & & \\
\cline { 2 - 3 } & $\begin{array}{l}\text { Energy } \\
\text { values }\end{array}$ & $\begin{array}{l}a^{*}=-1 \\
a \text { (present } \\
\text { model) }\end{array}$ & $\begin{array}{l}\text { Percent- } \\
\text { age error }\end{array}$ & \\
valn
\end{tabular}

\begin{tabular}{lll}
$E_{1,3 / 2}^{-}$ & & \\
\hline $\begin{array}{l}\text { Energy } \\
\text { values }\end{array}$ & $\begin{array}{l}a^{*}=3 \\
a \text { (present } \\
\text { model) }\end{array}$ & $\begin{array}{l}\text { Percent- } \\
\text { age } \\
\text { error }\end{array}$
\end{tabular}

$14 \quad-0.2174 \quad-0.8521 \quad 0.8 \%$

$(-0.2157)$

$[-0.1870]$

$[-0.1870]$ [-0.2152]*

error

$\begin{array}{llll}19-0.4737 & -0.8944 & 0.28 \%\end{array}$

$(-0.4724)$

$[-0.4237]$

$\begin{array}{lll}-0.2170 & 3.1285 & 1.07 \%\end{array}$

$(-0.2147)$

$[-0.1870]$

$[-0.2150] * \quad 0.14 \%$

$\begin{array}{lll}-0.4720 & 3.1202 & 0.75 \%\end{array}$

$(-0.4685)$

$[-0.4237] *$

$24-0.8534 \quad-0.9095 \quad 0.13 \%$

$-0.8484$

$(-0.8545)$

$[-0.7810]$

$(-0.8438)$

$[-0.7810]$

$1.25 \%$

$[-0.8608]^{*}$

$-1.3544$

$(-1.349)$

$(-1.373)$

$[-1.2677]$

$-0.9195 \quad 0.51 \%$

$[-1.2677]$

$0.42 \%$

$34-2.0195$

$-0.9251 \quad 0.96 \%$

$(-2.039)$

[-1.8900]

$-1.9962$

$(-1.989)$
$[-1.8900]$

$0.62 \%$

[-2.0042]

$39-2.8212$

$-0.9277 \quad 1.35 \%$

$-2.7787$

$(-2.771)$

$(-2.860)$

$[-2.6531]$ [

$0.51 \%$

[-2.6531]

$74-13.2111 \quad-0.8998 \quad 5.72 \%$

$(-14.0123)$

[-12.2675]

$[-13.5045]^{*}$

$-12.5254$

$(-12.512)$

$[-12.2675]$

$79-15.4874-0.8904 \quad 6.72 \%$

$[-12.8811] *$

$-14.571$

$(-16.603)$

$[-14.2949]$

$(-14.557)$

$[-14.2949]$

$4.5 \%$

$84-17.9967$

$(-19.570)$

$[-16.4969]$

$-0.9099 \quad 8.04 \%$

$-16.7914$

(-16.776)

$[-16.4969]$

$1.15 \%$

$3.0842 \quad 0.54 \%$

$[-18.4767]^{*}$

$5.59 \%$

$[-17.4032] *$

\section{$2.01 \%$}

$3.0713 \quad 0.40 \%$

$1.42 \%$

$3.0617 \quad 0.36 \%$

$0.76 \%$

$3.0542 \quad 0.28 \%$

.

$1.26 \%$

$0.19 \%$

$2.95 \%$

$0.096 \%$

$3.20 \%$

$0.092 \%$

$3.74 \%$

Note: $a^{*}$ is the shift obtained from the formula $a^{*}=-(2 n+2 s-1)$. 


\section{Acknowledgement}

We are thankful to Mr. Prasanta Chatterjee of Physics and Applied Mathematics Unit, Indian Statis- tical Institute, Calcutta, for helping in numerical computation.

We are also grateful to the referee for very useful suggestions and comments.
[1] S. S. Stepanov and R. S. Tutik, Phys. Letters A 163, 26 (1992). - Zh. Exp. Theeor. Phys. 100, 415 (1991); J. Phys. 25, L 413 (1992).

[2] A. Atag, J. Math. Phys. 30, 696 (1989).

[3] R. Roychoudhury and Y. P. Varshni, J. Phys. A 20, L 1083 (1987).

[4] M. M. Panja and R. Dutta, Phys. Rev. A 38, 3937 (1988).

[5] R. Roychoudhury and Y. P. Varshni, Phys. Rev. A 37, 5523 (1989).
[6] A. Chatterjee, J. Math. Phys. 27, 2331 (1986).

[7] U. Sukhatme and T. Imbo, Phys. Rev. D 28, 416 (1983).

[8] A. Chatterjee, Phys. Rep. 186, 249 (1990).

[9] C. H. Mehta and S. H. Patil, Phys. Rev. A 17, 34 (1978).

[10] R. Pratt and H. Tseng, Phys. Rev. A 9, 1063 (1972).

[11] R. L. Hall, J. Math. Phys. 25, 2708 (1984); J. Phys. A 19, 2079 (1986).

[12] M. M. Panja, R. Dutta, and Y. P. Varshni, Phys. Rev. D 42, 106 (1990). 